\title{
PROJEÇÕES NOS FATOS RELEVANTES DAS EMPRESAS DE CAPITAL ABERTO DA B3: IMPACTOS DA COVID-19
}

\author{
PROJECTIONS IN RELEVANT FACTS OF B3 PUBLIC FIRMS: COVID-19 IMPACTS
}

\author{
MAYARA BEZERRA BARBOSA \\ Doutoranda em Ciências Contábeis - Universidade de Brasília (UnB) \\ Orcid: https://orcid.org/0000-0002-4620-3415 / E-mail: mayarabezerrab@yahoo.com.br \\ Av. dos Portugueses, s/n - São Luís/MA, CEP: 65080-805 \\ CÉSAR AUGUSTO TIBÚRCIO SILVA \\ Doutor em Controladoria e Contabilidade - Universidade de São Paulo (USP) \\ Orcid: https://orcid.org/0000-0002-5717-9502/ E-mail: cesaraugustotiburciosilva@gmail.com \\ MARIANA PEREIRA BONFIM \\ Doutora em Ciências Contábeis - Universidade de Brasília (UnB) \\ Orcid: https://orcid.org/0000-0003-2339-0462 / E-mail: marianapbonfim@gmail.com

\section{JOSÉ MAURO MADEIROS VELÔSO SOARES} \\ Doutorando em Ciências Contábeis - Universidade do Vale do Rio dos Sinos (Unisinos) \\ Orcid: https://orcid.org/0000-0002-9277-8316 / E-mail: jose-mauro-m@hotmail.com \\ Submissão: 15/08/2020. Revisão: 16/11/2020. Aceite: 19/12/2020. Publicação: 29/12/2020. \\ DOI: http://dx.doi.org/10.22277/rgo.v14i1.5747
}

\section{RESUMO}

As dimensões do efeito da COVID-19 e suas respectivas implicações nos negócios ainda é pouco conhecida. Para compreender aspectos relacionados a esse novo cenário, esta pesquisa teve por objetivo analisar os reflexos dos primeiros meses da pandemia da COVID-19 no volume de divulgação de fatos relevantes relacionados com as projeções das empresas de capital aberto da Brasil, Bolsa, Balcão (B3). Utilizou-se o banco de dados da Comissão de Valores Mobiliários (CVM), onde foram identificados 1.712 fatos relevantes (FR) divulgados entre $01 / 01 / 2020$ e 31/07/2020, além de $1.421 \mathrm{FR}$ para igual período em 2019. Excluindo os cancelados e inativos, foram lidos e analisados 2.882 (1.582 em 2020 e 1.300 em 2019) fatos relevantes, a fim de identificar a existência de conteúdo sobre projeções. A amostra final compreendeu, respectivamente em 2019 e 2020, 81 e 163 fatos relevantes, de 48 e 95 empresas, que mencionaram um dos termos: "previsão", "projeção", "estimativa" e/ou "guidance". Comparando com o mesmo período, houve um aumento de, aproximadamente, $33 \%$ na divulgação de fatos relevantes em 2020, destacando-se os relacionados à suspensão de projeções de atividades e de previsões de resultados. O setor financeiro e o de transportes foram os que mais divulgaram fatos no período, ainda que de forma não tempestiva. Esta pesquisa contribui para a reflexão e avaliação de melhorias no processo de previsões da informação contábil, com observância ao comportamento e tempestividade dos gestores em reportar fatos relevantes que possam impactar a tomada de decisão dos usuários.

Palavras-chave: Projeções. Fatos relevantes. Tempestividade. COVID-19. Coronavírus. 
Mayara Bezerra Barbosa, César Augusto Tibúrcio Silva, Mariana Pereira Bonfim e José Mauro Madeiros Velôso Soares

\begin{abstract}
The dimensions of the COVID-19 effect and its implications for business are still poorly understood. To understand aspects related to this new scenario, this research aimed to analyze the reflections of the first months of the COVID-19 pandemic in the volume of disclosure of relevant facts related to the projections of publicly traded companies in Brazil, Bolsa, Balcão (B3). The Securities and Exchange Commission (CVM) database was used, identifying 1,712 relevant facts (FR) disclosed between 01/01/2020 and 07/31/2020, in addition to 1,421 FR for the same period in 2019. Excluding the canceled and inactive, 2,882 (1,582 in 2020 and 1,300 in 2019) relevant facts were read and analyzed to identify the existence of content about projections. The final sample comprised, respectively in 2019 and 2020, 81 and 163 relevant facts, from 48 and 95 companies, which mentioned one of the terms: "forecast," "projection", "estimate", and/or "guidance". Compared to the same period, there was an increase of approximately 33\% in the disclosure of relevant facts in 2020, emphasizing those related to the suspension of projections of activities and forecasts of results. The financial and transport sectors were the ones that most disclosed facts in the period, albeit in a non-timely manner. This research contributes to the reflection and evaluation of improvements in the forecasting process of accounting information, with the observance of managers' behavior and timing in reporting relevant facts that may impact users' decision-making.
\end{abstract}

Keywords: Projections. Relevant fact. Timeliness. COVID-19. Coronavirus.

\title{
1 INTRODUÇÃO
}

Em dezembro de 2019, foi relatado o primeiro caso de COVID-19 na China e, no início do ano de 2020, e em decorrência do seu alto poder de contágio, a doença já havia se espalhado por diversos países, inclusive o Brasil, tendo a Organização Mundial de Saúde (OMS) decretado, em março de 2020, o surto como pandemia. Com a rápida transmissão do vírus, a falta de capacidade dos sistemas de saúde para suportar a quantidade de doentes e a inexistência de vacinas e tratamentos específicos, a solução adotada por governos foi a suspensão das atividades econômicas e sociais da população.

Em um mundo fortemente conectado e integrado, os impactos da doença, para além da mortalidade, se tornaram evidentes: a desaceleração da economia das principais potências mundiais, interrupção da produção de bens considerados não essenciais e quedas nos mercados financeiros globais são algumas das consequências da COVID-19 (McKIBBIN; FERNANDO, 2020). Por meio do avanço da doença, a crise se espalhou por outros países em função da interdependência econômica entre eles (SINGH; SINGH, 2017).

A interdependência relaciona-se com a existência de vínculos que correlacionam os países em relação a acontecimentos com impacto econômico (FORBES; RIGOBON, 2002; ISMAILESCU; KAZEMI, 2011). Desse modo, os impactos econômicos e financeiros no Brasil, ocasionados em virtude da pandemia, também se fazem presentes em função da existência da interdependência econômica brasileira como parceira comercial e de investimento com diversos países, e expressivamente relevante com a China (GALE; VALDES; ASH, 2019; GOUVEA; MONTOYA, 2013; GOUVEA; KAPELIANIS; LI, 2020; WORLD BANK, 2018).

Diante da pior crise econômica desde a grande Depressão (IFM, 2020) e com base em Dovern e Janssen (2017), espera-se que as previsões prévias superestimem o crescimento do Produto Interno Bruto (PIB) e estão sujeitas a grandes erros sistemáticos negativos. Os 
Projeções nos fatos relevantes das empresas de capital aberto da B3:

impactos da COVID-19

impactos nas empresas podem ser semelhantes, uma vez que os modelos de previsão se baseiam fortemente em dados históricos e existe excesso de confiança nas previsões emitidas (HRIBAR; YANG, 2015). No âmbito das questões epidemiológicas, loannidis, Cripps e Tanner (2020) destacam a admissibilidade de que as previsões relacionadas as ações e aos impactos para a tomada de decisões sejam falhas, destacando a insuficiência de dados e evidências anteriores para que se tenha mais acurácia.

A contabilidade deve retratar com fidedignidade a situação econômico-financeira das empresas. Assim, os efeitos da pandemia da COVID-19, no qual empresas interromperam suas atividades ou adaptaram-se em trabalhos remotos, devem ser reconhecidas e divulgadas aos usuários (DELOITTE, 2020). loannidis (2020) destaca que o cenário no qual estavam sendo tomadas as decisões era permeado de incerteza, inerente à falta de precedentes, e informações confusas sobre o impacto e o vírus em si. Neste sentido, a divulgação de fatos relevantes permite às empresas sinalizarem as medidas tomadas em resposta ao presente cenário e preservar a confiança aos principais usuários.

Diante do exposto, surge o seguinte problema de pesquisa: Quais são os reflexos dos primeiros meses da pandemia da COVID-19 na divulgação de fatos relevantes, relacionados às projeções, das empresas brasileiras de capital aberto da Brasil, Bolsa, Balcão (B3)? Desse modo, o estudo tem como objetivo analisar os reflexos dos primeiros meses da pandemia da COVID-19 no volume de divulgação de fatos relevantes relacionados com as projeções das empresas de capital aberto da B3. Para atingir este objetivo, todos os fatos relevantes divulgados entre $01 / 01 / 2020$ e 31/07/2020 comparativamente com o mesmo período de 2019 (01/01/2019 e 31/07/2019), foram coletados, manualmente, e lidos para identificação do conteúdo relacionado às projeções.

A CVM estabelece, por meio da Instrução CVM n 358, de 2002, que sejam divulgados como fatos relevantes, dentre outros, os eventos econômico-financeiros, tanto que tenham ocorrido, como que estejam relacionados aos negócios das empresas e possam influenciar na cotação, na decisão de investidores, ou no exercício dos seus direitos. Contudo, embora a legislação estabeleça que a informação tenha que ser clara, precisa, e em linguagem acessível, há certa discricionariedade na divulgação, em razão de ser realizada pela própria empresa e potencialmente impactar nas percepções sobre ela mesma.

A literatura prévia sobre o conteúdo dos fatos relevantes demonstra que, em geral, as divulgações possuem baixa legibilidade (SILVA; FERNANDES, 2009), ou tem legibilidade variável de acordo com o evento reportado, sendo mais fácil quando positivo e mais difícil quando negativo (BERNARDES et al., 2018). Entretanto, por mais que tratem de informações tempestivas com linguagem pessimista ou otimista, o tom de sua divulgação não exerceu, no passado, influência significativa nos preços das ações (SILVA; FELIPE, 2010; BERNARDES et al., 2018).

A presente pesquisa difere-se das demais pelo período, entre 2019 e 2020, critério de identificação dos fatos relevantes que tratam de projeções, e método de análise quantitativo e qualitativo, além de abordar a ocorrência de um evento pandêmico com impacto geral para todas as empresas e com um alcance desconhecido e incerto no momento da sua necessidade de divulgação. Sendo assim, contribui com a literatura ao analisar o conteúdo dos discursos das empresas nos fatos relevantes divulgados em relação ao impacto da pandemia do novo coronavírus.

Esta análise é importante, pois permite entender algumas nuances importantes do processo de comunicação de uma empresa, em um sentido lato, diante de um evento extremo, como ocorreu com a pandemia. Em um sentido restrito, destaca uma discussão 
Mayara Bezerra Barbosa, César Augusto Tibúrcio Silva, Mariana Pereira Bonfim e José Mauro Madeiros Velôso Soares

sobre a reação dos agentes econômicos sobre sua visão de futuro, diante de um acontecimento externo, com implicações para todas as empresas. Trata-se de um recorte temporal específico, em uma situação de incerteza, e como as empresas reagiram e evidenciaram para os usuários. Neste sentido, há uma contribuição teórico-prática para a literatura sobre divulgações de informações financeiras e em relação ao conteúdo dos fatos relevantes.

Os resultados da pesquisa mostram um aumento no número de fatos relevantes no período após a declaração da pandemia, representando mais informação para ser processada pelo usuário. Mesmo quando a empresa não divulga um fato relevante, indicando que as estimativas realizadas no ambiente anterior à pandemia não são mais válidas, esta "ausência" de um fato relevante provavelmente irá aumentar a incerteza do usuário quanto aos efeitos da pandemia nas atividades da empresa.

\section{REFERENCIAL TEÓRICO}

\subsection{TEORIA DA DIVULGAÇÃO}

A divulgação de informações de natureza econômica e financeira pelas companhias é importante para os usuários tomarem decisões, tanto no que diz respeito à previsão de fluxos de caixa quanto para a avaliação do uso dos recursos econômicos (NIYAMA, SILVA, 2013; ARGILÉS-BOSCH et al., 2018; CPC, 2019). Por isso, é imprescindível que as empresas divulguem suas informações, sejam elas qualitativas ou quantitativas, com qualidade e de forma tempestiva (SILVA; SOUZA; KLANN, 2016). Entretanto, os usuários podem necessitar de informações além daquelas obrigatórias; por isso, divulgar dados de forma voluntária pode ser uma ferramenta útil para expressar a realidade da empresa (BROWN, 2020).

A informação compulsória pode ser entendida como aquela que provém de medidas legais, impondo aos administrados a sua divulgação. Esse mecanismo possibilita uma redução da assimetria informacional, sob penas jurídicas contra a empresa que não o realizar. Por outro lado, as informações voluntárias são aquelas que excedem as exigências legais, representando uma livre escolha (free choice) dos gestores em divulgá-la ou não. Importante ressaltar que as divulgações voluntárias não são responsáveis por eliminar as distorções entre agente e principal (DANTAS et al., 2005).

Brown (2002, p. 189) cita os cinco maiores benefícios, identificados por analistas e investidores, de uma melhor evidenciação: "aumento da credibilidade dos gestores, maior monitoramento por parte dos analistas, melhor avaliação do preço das ações, mais investidores de longo prazo e diminuição do custo de capital". Ou seja, uma maior transparência da empresa na divulgação de informações afeta a sua performance econômica.

Verrecchia (2001) destaca que não existe uma teoria da divulgação unificada, mas realiza uma categorização dos modelos utilizados na Contabilidade e propõe a seguinte taxonomia para as pesquisas sobre divulgação: a) baseada em associação (association-based disclosure); b) baseada em julgamento (discretionary-based disclosure) e; c) baseada em eficiência (efficiency-based disclosure). A primeira categoria compreende pesquisas que buscam associar a divulgação e as mudanças no comportamento dos investidores (processo exógeno), ou seja, de que forma a divulgação modifica a ação dos investidores. A segunda categoria engloba pesquisas que compreendam o julgamento dos gestores em divulgar ou não uma informação (processo endógeno), considerando os incentivos que os mesmos recebem. Por fim, a última categoria classifica as pesquisas que discutem quais os tipos de divulgação são mais eficientes. 
Projeções nos fatos relevantes das empresas de capital aberto da B3: impactos da COVID-19

Desse modo, a presente pesquisa alinha-se com mais proximidade à utilização da teoria da divulgação no âmbito de associações, entre características do ambiente ou das empresas, e a evidenciação de informações. Ainda que seja presumido que a informação divulgada no fato relevante seria aquela cujo conhecimento prévio não se dá por outro documento (efficiency-based), ao associar a pandemia da COVID-19 com possíveis reflexos na divulgação de fatos relevantes relacionados às projeções, esse estudo traz a perspectiva do disclosure baseado em associação, especialmente quando compara com as divulgações realizadas no ano anterior.

Trabalhos anteriores verificaram associação de divulgação voluntária com o desempenho (SALOTTI; YAMAMOTO, 2008), preferência em divulgação de informações positiva em detrimento das negativas (ROVER; BORBA; MURCIA, 2009) e ocorrência de retornos anormais para as companhias associados à divulgação de eventos subsequentes (KOS; BARROS; COLAUTO, 2017). Rover et al. (2008) destacam que informações voluntárias beneficiam as empresas por meio de redução do custo de capital e aumento na liquidez das ações.

\subsection{FATOS RELEVANTES}

Diante da necessidade de divulgação de informações tempestivas para a tomada de decisão dos usuários e dos benefícios do disclosure voluntário, a publicação de fatos relevantes representa uma importante ferramenta de comunicação entre a empresa e o mercado (SENA; SILVA; ARRIAL, 2010). Expressos em textos narrativos e, geralmente, não auditados, os fatos relevantes são considerados de divulgação voluntária, auxiliando na redução da assimetria de informações e reduzindo a existência de informações privilegiadas (SILVA; FERNANDES, 2009).

Ao contrário das demonstrações financeiras, que são divulgadas em períodos específicos e com uma regularidade associada a um horizonte temporal, os fatos relevantes não possuem esta limitação. Além disso, enquanto as demonstrações financeiras possuem um rito processual, passando pelo processo de auditoria, diferentes comitês e conselhos, o fato relevante pode ser divulgado de maneira mais ágil, tão logo seja necessário. Em outras palavras, o tempo entre o evento contábil/administrativo e sua divulgação é muito curto, ao contrário das demonstrações financeiras (SILVA; FERNANDES, 2009). Outra distinção é que o fato relevante geralmente faz uso da linguagem escrita, permitindo uma análise mais rica do acontecimento que é retratado.

As empresas brasileiras, atendendo a Instrução CVM no 358/2002, devem apresentar nos fatos relevantes os acontecimentos de forma tempestiva, relacionados às projeções financeiras e operacionais, avaliação de riscos e incertezas do negócio, estabelecendo premissas de crescimento e divulgando atos ou fatos de caráter político-administrativo, técnico, negocial ou econômico-financeiro ocorrido ou relacionado aos seus negócios. 0 objetivo é melhorar a comunicação da empresa com os principais usuários da informação contábil, visando a tempestividade, e possibilitando a esses conhecerem as perspectivas futuras da firma (CEN; CAl, 2013).

A divulgação de relatórios tempestivos, principalmente quando tratam sobre os riscos associados aos negócios, contribuem para a redução da assimetria informacional, sendo útil para a tomada de decisão dos investidores (MIIHKINEM, 2013). Santos e Coelho (2018) verificaram que os fatores de riscos mais presentes nos relatórios das empresas brasileiras são concorrência, desenvolvimento tecnológico, instabilidade política, situação econômica 
Mayara Bezerra Barbosa, César Augusto Tibúrcio Silva, Mariana Pereira Bonfim e José Mauro Madeiros Velôso Soares

mundial e escassez de recursos. A pandemia da COVID-19 trouxe um impacto significativo na economia mundial, ocasionada pela paralisação das atividades em diversos setores, desencadeando em consequência, na escassez de recursos. A instabilidade política é um fator identificado não só no Brasil, como em toda a América Latina (LIMA; BUSS; PAES-SOUSA, 2020), tendo sido aprofundado com a pandemia. Dessa forma, a divulgação desses fatores de risco por parte das empresas se torna importante na tomada de decisão de investidores, especialmente se realizados de forma tempestiva, o que é possível através dos fatos relevantes.

A Instrução 358 da CVM (2002) explicita o dever de divulgação dos atos ou fatos relevantes, de forma clara e precisa, como forma de fazer com que o mercado conheça as informações que impactam as operações e seus respectivos reflexos nas atividades. Este aspecto é reafirmado e reiterado em notícias e comunicados feitos ao mercado oficialmente. A exemplo disto, a CVM, por meio do Ofício Circular 02/2016, direcionado aos Diretores de Relações com Investidores, reitera a preocupação em fazer com que os atos ou fatos relevantes sejam publicados, de forma prévia ou simultânea, antes de serem discutidos em quaisquer reuniões com investidores, analistas ou qualquer grupo que se equipare, como forma de garantir equidade no acesso à informação e mitigar problemas de assimetria informacional. Sendo assim, espera-se que estes documentos sejam a divulgação mais tempestiva e o documento hábil a influenciar na tomada de decisão dos stakeholders.

A relevância e a legibilidade das informações divulgadas em fatos relevantes das empresas são apontadas por Silva e Fernandes (2009) e Bernardes et al. (2018), sendo considerada importante a tempestividade e o comportamento dos administradores, de maneira otimista ou pessimista, presente nestes relatórios. No atual cenário, as premissas operacionais e de continuidade devem ser divulgadas, considerando que não se conhece o término da crise decorrente da COVID-19 (DENNIS; SEERY, 2020).

Pesquisas anteriores que se utilizaram dos fatos relevantes evidenciaram que, em geral, os textos são de difícil compreensão por parte dos usuários. Silva e Fernandes (2009) analisaram o nível de legibilidade dos fatos emitidos entre 2002 e 2006, através do Método de Flesch, e verificaram que somente $10 \%$ dos textos são de fácil leitura. Os fatos emitidos nesse período sobre projeções foram considerados, pelos autores, como de difícil legibilidade. Analisando o mesmo período, Espíndula e Costa (2008) verificaram que os fatos relevantes vêm aumentando de tamanho ao longo do tempo e que apenas no setor de petróleo e gás, a divulgação e a legibilidade causam impacto no preço das ações.

Ferreira (2010) analisou não só o impacto dos fatos relevantes nos preços das ações como também as notícias divulgadas na imprensa e verificou que a imprensa divulga mais do que os fatos relevantes. Após o dia de elevada negociação das ações no mercado, as notícias mais divulgadas pelas empresas foram sobre o lucro ou prejuízo da empresa e sobre projeções ou mudanças nas previsões da sua atividade. Diante de um grande impacto no mercado financeiro brasileiro, inclusive com a ocorrência de seis circuit breaker na B3, é esperado que a divulgação de informações a respeito das projeções se intensifique no período inicial da pandemia.

Bernardes et al. (2018) analisaram o discurso presente nos textos narrativos da Vale S.A., focando no período após o rompimento da barragem do Fundão, em Mariana, Minas Gerais. Através da técnica de análise do discurso, os autores verificaram que a companhia, em um cenário pessimista, apresentou fatos relevantes com menor legibilidade, se comparado com os publicados em cenários otimistas. Após a queda da barragem, os fatos publicados 
Projeções nos fatos relevantes das empresas de capital aberto da B3: impactos da COVID-19

buscavam, preponderantemente, acalmar o mercado quanto à possibilidade de ações cíveis e evitar esclarecimentos sobre o ocorrido.

Semelhantemente, Marques et al. (2010) identificaram que $88 \%$ dos fatos relevantes emitidos pelas empresas brasileiras retratam "boas notícias", e que apenas $5 \%$ foram "más notícias". De forma similar, diante de um cenário pessimista como o da pandemia da COVID19 , é esperado que as empresas divulguem os fatos relevantes tranquilizando os investidores em relação à incerteza inerente ao momento e como a empresa é afetada pelos eventos ocorridos. Diante destes apontamentos, faz-se necessário conhecer o conteúdo dos fatos relevantes após a pandemia para assim analisar quais são os reflexos dos primeiros meses da pandemia da COVID-19 nestas divulgações, no que está relacionado às projeções, tendo em vista que há uma pressuposição de que o evento tem potencial impacto em todas as empresas em função de sua amplitude, ainda que possa afetar cada uma de uma forma diferente.

\section{PROCEDIMENTOS METODOLÓGICOS}

Para proceder com a análise das evidências foi realizada uma pesquisa descritiva de natureza documental. Como unidade de análise, foram utilizados todos os fatos relevantes das empresas de capital aberto que estavam disponíveis no site da CVM (www.cvm.gov.br), por meio da utilização do sistema de Recebimento Automático de Documentos (RAD).

O período de publicação dos documentos analisados foi do dia 01/01/2020 até $31 / 07 / 2020$, comparativamente com igual período do ano anterior. Com relação aos fatos de 2020, os mesmos foram coletados no período entre abril e julho. Ao todo, foram publicados 1.712 fatos relevantes até $31 / 07 / 2020$, sendo 116 classificados como inativos e 14 cancelados, portanto, teve-se acesso à 1.582 documentos. Para o período de 01/01/2019 à 31/07/2019, foram identificados 1.421 fatos relevantes, sendo 102 inativos e 19 cancelados. Os dados de 2019 foram coletados entre 18 a 24 de novembro de 2020.

Para a constituição do corpus, foram selecionados os fatos que possuíam as expressões "previsão", "projeção", "estimativa" e "guidance", no singular e plural dessas palavras. Do total de fatos divulgados no período, foram identificadas as palavras em 244 documentos, constituindo o material a ser analisado.

A partir da leitura dos fatos relevantes publicados pelas companhias abertas brasileiras, buscou-se interpretar sua mensagem, especialmente no que tange às previsões. Partindo da premissa de que não é possível estabelecer uma leitura neutra, pois toda leitura se constitui uma interpretação, adotou-se, portanto, a análise de conteúdo, que se utiliza de técnicas para se alcançar as mesmas interpretações. Segundo Bardin (2016), a análise de conteúdo pode ser entendida como uma técnica que se utiliza de instrumentos metodológicos sistemáticos e objetivos, de descrição do conteúdo da mensagem, de modo a inferir conhecimentos relativos às condições da mensagem.

Todos os fatos relevantes foram coletados, para serem lidos e identificados em relação ao tópico por meio de navegação lexical como critério de inclusão. A coleta de todos os documentos e acesso individual a cada registro tem por objetivo identificar, de forma mais criteriosa, se o tópico sob investigação foi tratado ou não. Dos fatos relevantes que apresentavam os critérios de inclusão, foram excluídos os fatos que não apresentavam os conteúdos no corpo do texto, mas nas notas de rodapé, relatando que as informações divulgadas poderiam conter estimativas e projeções da administração. A leitura dos fatos possibilitou identificar casos em que palavras de mesmo radical ou grafia eram utilizadas em situações adversas ao escopo de projeções desta pesquisa. 
Mayara Bezerra Barbosa, César Augusto Tibúrcio Silva, Mariana Pereira Bonfim e José Mauro Madeiros Velôso Soares

De forma exemplificativa para uma breve comparação, caso não fossem coletados todos os arquivos do período, um a um, e procedido à leitura, identificar-se-ia no sistema da CVM, pelo seu mecanismo de busca por meio das palavras-chave, 71 registros, em vez de 163 (129,57\% maior), para o ano de 2020 . Os dados desta pesquisa estão registrados publicamente na plataforma Mendeley (data.mendeley.com) e podem ser acessados por meio do seu Identificador Digital de Objeto: 10.17632/g75hrsszb9.1.

\section{RESULTADOS}

Em 2019, identificou-se 48 empresas que emitiram 81 fatos relevantes mencionando ao menos um dos termos em análise e, em 2020 foram 95 empresas que divulgaram 163 fatos relevantes sobre projeções. As empresas Gol Linhas Aéreas Inteligentes S.A. e a Vale S.A. foram as que mais divulgaram sobre o tema em 2019, tendo 6 FR cada uma, e em 2020 foi a Vale S.A., com 10 fatos. Observou-se também que em 2019 e 2020, respectivamente, outras 28 e 50 empresas divulgaram somente um fato relevante relacionado ao tema. Na Tabela 1 apresentam-se os resultados das quantificações dos dados.

Tabela 1 - Número de FR com os termos selecionados

\begin{tabular}{c|c|c|c|c}
\hline Período & Previsão & Projeção & Estimativa & Guidance \\
\hline Até julho de 2019 & 5 & 32 & 42 & 37 \\
Até julho de 2020 & 51 & 88 & 83 & 55 \\
\hline
\end{tabular}

Fonte: elaborada pelos autores, com base nos dados da pesquisa (2020).

A partir dos dados da pesquisa é possível observar que os termos pesquisados foram citados 1.557 vezes, com uma média de 6,38 termos por documento. 0 termo mais utilizado foi "projeção/projeções", sendo citado, em média, 4,43 por fatos relevantes identificados da amostra, e o termo menos citado foi "previsão", aparecendo, em média, 1,63 vezes nos fatos relevantes.

Na Tabela 2 é apresentada a distribuição dos fatos relevantes por setores, visando apresentar quais setores tiveram maior número de emissões de FR pelas empresas que o compõem, e buscando compreender o comportamento comparativo ao período correspondente do ano anterior.

Tabela 2 - Frequência de fatos relevantes por setores - janeiro a julho de cada ano

\begin{tabular}{clcc}
\hline \multicolumn{1}{c}{ Setores } & Qtd de FR 2020 & Qtd de FR 2019 \\
\hline 1. & Financeiro & 25 & 19 \\
2. & Outros & 25 & 12 \\
3. & Transporte & 22 & 13 \\
4. & Petróleo, Gás e Biocombustíveis & 13 & 6 \\
5. & Energia Elétrica & 12 & 1 \\
6. & Comércio & 11 & 2 \\
7. & Construção, Engenharia e Construção Civil & 9 & 2 \\
8. & Agropecuária e Alimentos & 8 & 9 \\
9. & Tecidos, Vestuário e Calçados & 8 & 3 \\
10. & Comunicações & 5 & 5 \\
11. & Siderurgia e Metalurgia & 5 & 7 \\
12. & Viagens e Lazer & 5 & - \\
13. & Água, Saneamento e Gás & 3 & 2 \\
14. & Alimentos Processados & 3 & - \\
15. & Saúde & 3 & - \\
16. & Máquinas e Equipamentos & 2 & - \\
17. & Materiais Básicos & 2 & - \\
18. & Tecnologia da Informação & 2 & $\mathbf{8 1}$ \\
\hline
\end{tabular}

Fonte: elaborada pelos autores, com base nos dados da pesquisa (2020). 
Projeções nos fatos relevantes das empresas de capital aberto da B3:

impactos da COVID-19

O setor financeiro apresentou o maior número de fatos relevantes em ambos os períodos, totalizando 25 em 2020 e 19 em 2019, resultado esse que pode ser explicado pelas peculiaridades de suas atividades e os riscos inerentes ao setor relativos à períodos de crises e recessões. Em sequência, os dados evidenciam que em 2020, os setores de transportes divulgaram 22 fatos relevantes relacionados a projeções, assim como as empresas de petróleo, gás e biocombustíveis (13); energia elétrica (12) e comércio (11), conforme Tabela 2. Esses 5 setores juntos apresentaram $51 \%$ dos fatos relevantes de 2020 relacionados às projeções no período.

Esses resultados corroboram informações divulgada pela Deloitte (2020) no qual previa possíveis setores que seriam afetados, em menor ou maior escala, pela crise causada pela COVID-19, em decorrência de muitas empresas terem interrompidos suas atividades ou adaptaram-se para trabalhos remotos (home office).

Com relação ao ano de 2020, é importante destacar que 7 dos 9 fatos divulgados pelas empresas do setor de construção, engenharia e construção civil referem-se à suspensão das projeções previstas, seja de ofertas de ações ou das atividades operacionais. Na composição de "outros" destaca-se os subsetores de mineração (11), madeira e papel (4), sendo todos esses divulgados pela empresa Suzano S.A., e o subsetor de diversos (7), composto pelos segmentos de aluguel de carros, programas de fidelização e serviços educacionais. Ressaltase também que os setores de alimentos processados, saúde, viagens e lazer, máquinas e equipamentos, materiais básicos e tecnologia da informação apresentaram fatos relevantes sobre projeções em 2020, mas nenhum em 2019, o que pode indicar o efeito da pandemia nas companhias desse setor.

Em 30 de janeiro de 2020, a OMS declarou o surto da doença como Emergência de Saúde Pública de Importância Internacional. Em 10 de março, a CVM expediu o Ofício-Circular CVM/SNC/SEP n.. 02/2020 que trata de orientações sobre os efeitos do novo coronavírus nas demonstrações financeiras, destacando-se a necessidade de divulgação de fato relevante e de projeções e estimativas relacionadas aos riscos da doença. Em 11 de março, a COVID-19 foi caracterizada pela OMS como uma pandemia. A relação temporal entre a divulgação de informações em fatos relevantes das empresas e a COVID-19, no ano de 2020, são apresentados na Figura 1.

Figura 1 - Distribuição de frequência ao longo do tempo - 2020

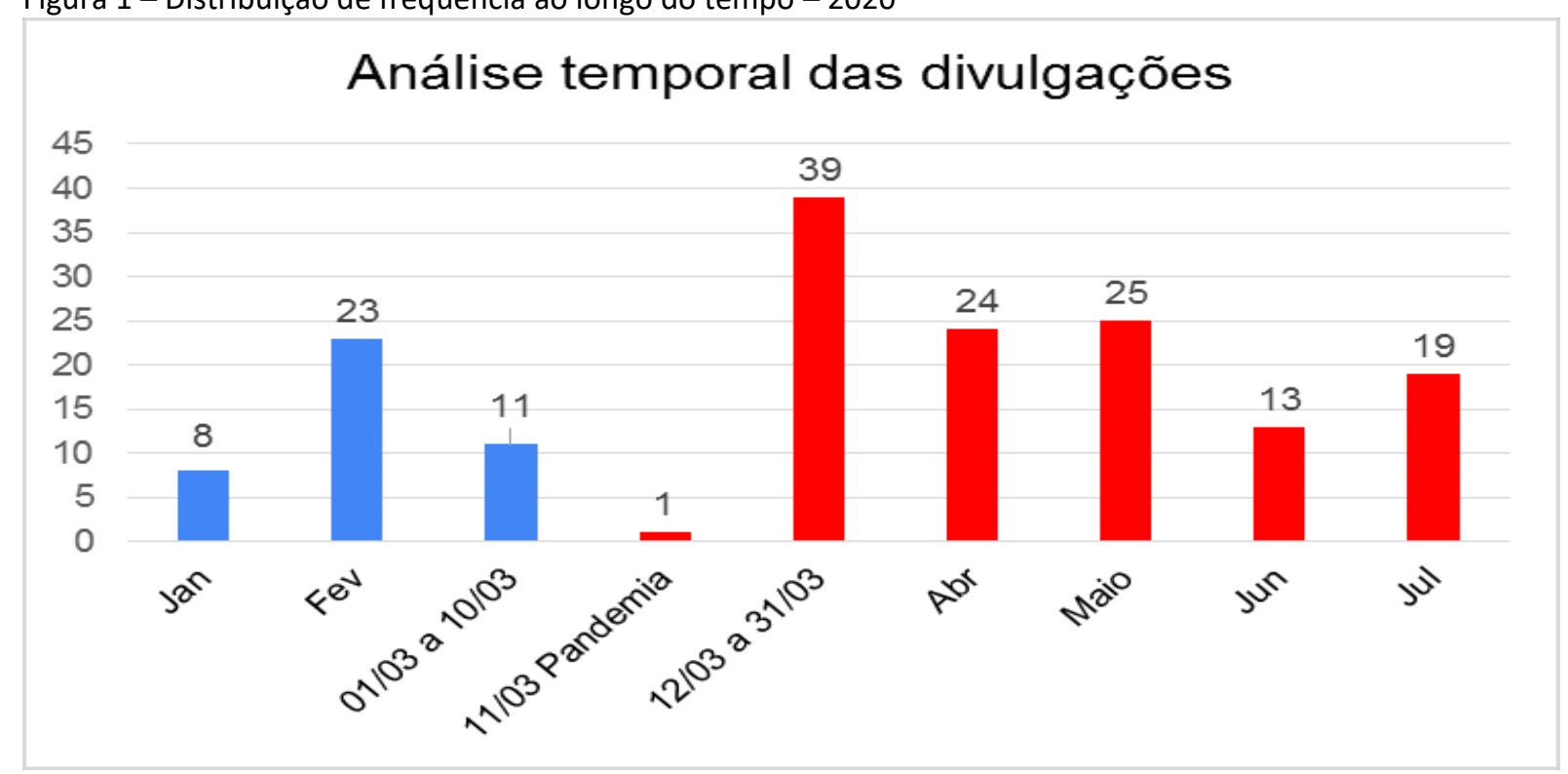

Fonte: elaborada pelos autores (2020). 
Mayara Bezerra Barbosa, César Augusto Tibúrcio Silva, Mariana Pereira Bonfim e José Mauro Madeiros Velôso Soares

Os resultados evidenciam que, no período imediatamente posterior a declaração da doença como pandemia pela OMS, houve um aumento nos fatos relevantes relacionados às projeções das empresas. No período de 01/01/2020 a 10/03/2020 foram divulgados 42 fatos relevantes, enquanto no período de 12/03/2020 a 31/07/2020 foram divulgados 120 , portanto, 78 fatos à mais do que no período anterior.

A análise da média de divulgação dos fatos relevantes relacionados às projeções realizada pela quantidade de dias padrão no período, evidenciada na Tabela 3, permite compreender o comportamento tempestivo da comunicação das empresas.

Tabela 3 - Média dos fatos relevantes

\begin{tabular}{l|c|cc|cc}
\hline \multicolumn{2}{c|}{ Período } & \multicolumn{2}{c|}{ 2019 } & \multicolumn{2}{c}{2020} \\
\hline & Quant. Dias & FR com projeções & Média diária & FR com projeções & Média diária \\
\hline Janeiro & 31 & 7 & 0,23 & 8 & 0,26 \\
Fevereiro & 28 & 27 & 0,96 & 23 & 0,82 \\
01/03 a 10/03 & 10 & 1 & 0,1 & 11 & 1,1 \\
$11 /$ mar & 1 & 0 & 0 & 1 & 1 \\
$12 / 03$ a 31/03 & 20 & 12 & 0,6 & 39 & 1,95 \\
Abril & 30 & 6 & 0,2 & 24 & 0,8 \\
Maio & 31 & 12 & 0,39 & 25 & 0,81 \\
Junho & 30 & 2 & 0,07 & 13 & 0,43 \\
Julho & 31 & 14 & 0,45 & 19 & 0,61 \\
\hline
\end{tabular}

Fonte: elaborada pelos autores (2020).

Em 11 de março de 2020, a empresa Enauta Participações S.A. apresentava suas projeções operacionais com validade de 1 ano, destacando que "a projeção representa uma estimativa baseada em crenças e premissas da Companhia e sujeita a diversos riscos e incertezas que podem não estar sob controle da administração", todavia nada menciona a respeito dos riscos relacionados a COVID-19 que já era uma realidade. A referida empresa só volta a atualizar suas projeções em 16 de julho do mesmo ano, reduzindo-as, sem mencionar os fatores que levaram as mudanças, assim como não faz referência a COVID-19.

Observa-se que a maior média diária de fatos relevantes ocorreu no período do mês de março de 2020, compreendendo os dias entre 11 a 31, sendo divulgados 1,95 fatos relevantes/dia sobre o assunto pesquisado. Nos períodos seguintes, de abril $(0,80)$ e maio $(0,81)$, manteve-se constante os números de divulgações. Em comparação, no período de 2020, janeiro apresentou a menor média diária de $\operatorname{FR}(0,26)$. Esses resultados, quando comparados com o exercício 2019, corroboram as evidências de impacto no aumento quantitativo de FR divulgados logo após a constatação da pandemia. O número de FR entre 12 e 31 de março de 2020 foram 3 vezes superiores ao quantitativo no mesmo período de 2019.

No ano de 2019, observou-se que, no período de 12 a 31 de março, 11 das 12 empresas analisadas estavam "comunicando" ou "informando" ao mercado suas projeções para o exercício, em cumprimento ao disposto na Lei $n^{\circ}$ 6.404, na Instrução CVM no 358 e na Instrução CVM n 480. Já em 2020, no mesmo período, foi divulgado em 22 dos 39 fatos publicados nesse período, suspensões das projeções anteriormente divulgadas, ou foi mencionado "não ser possível divulgar impactos da COVID-19".

As evidências apontam que a Paranapanema S.A. foi a primeira empresa a mencionar, no FR de 16/03/2020, que não seria possível ainda divulgar as incertezas e riscos relacionados à COVID-19, mas destaca que:

o avanço do novo coronavírus (COVID-19) pelo mundo têm provocado impactos negativos nos mercados globais e elevado as preocupações de investidores, sem

RGO - Revista Gestão Organizacional, Chapecó, v. 14, n. 1, p. 153-171, jan./abr. 2021. 
Projeções nos fatos relevantes das empresas de capital aberto da B3:

impactos da COVID-19

mencionar também as preocupações envolvendo as questões básicas de saúde da população mundial. Neste momento, não há como precisar os impactos a longo prazo no cenário econômico, e, em especial, os impactos nas operações da Companhia. Entretanto, tendo em vista a já observada diminuição da atividade econômica mundial, este cenário pode impactar o nível de atividade da Companhia (Paranapanema S.A.).

O setor financeiro impulsionou o aumento dos fatos relevantes em 2020, mas é importante destacar que 10 instituições financeiras divulgaram a suspensão de projeções somente a partir de 30/04/2020, 50 dias após a OMS ter decretado a COVID-19 como pandemia. O setor de transportes emitiu 18 fatos relevantes após 11/03/2020, todavia, 50\% destes ocorreram entre 24 de abril e 31 de julho, o que pode ser considerado não tempestivo aos cenários observados.

A frequência total de fatos relevantes divulgados pelas empresas, ocorridas entre as datas de 01 de janeiro a 31 de julho dos períodos de 2019 e 2020, permite inferir que ocorreu um efeito significativo na divulgação de fatos relevantes, sinalizando para o impacto causado pela COVID-19 nas divulgações das empresas, totalizando 82 FR a mais em comparação ao mesmo período no ano de 2019, representando um aumento total de $50,3 \%$. Considerando apenas os FR divulgados entre $11 /$ mar a $31 /$ jul de cada ano, observa-se um aumento de $32,6 \%$ no número total desse tipo de comunicação das empresas com o mercado. A Tabela 4 permite compreender a comparação entre o total de FR nos períodos e entre os relacionados às projeções.

Tabela 4 - Comparação dos fatos relevantes por ano

\begin{tabular}{lccccccc}
\hline Período & FR 2019 & FR 2020 & A por período & $\begin{array}{c}\text { FR com } \\
\text { projeções } \\
\mathbf{2 0 1 9}\end{array}$ & $\begin{array}{c}\mathbf{2 0 1 9 \%} \\
\begin{array}{c}\text { FR com } \\
\text { projeções } \\
\mathbf{2 0 2 0}\end{array}\end{array}$ & $\mathbf{2 0 2 0 \%}$ \\
\hline 01/01 a 10/03 & 381 & 365 & -16 & 35 & $9 \%$ & 42 & $12 \%$ \\
$11 / 03$ & 11 & 13 & 2 & 0 & $0 \%$ & 1 & $8 \%$ \\
$12 / 03$ a 31/03 & 908 & 1.204 & 296 & 46 & $5 \%$ & 120 & $10 \%$ \\
\hline & $\mathbf{1 . 3 0 0}$ & $\mathbf{1 . 5 8 2}$ & $\mathbf{2 8 2}$ & $\mathbf{8 1}$ & & $\mathbf{1 6 3}$ \\
\hline
\end{tabular}

Fonte: elaborada pelos autores (2020).

Relativo ao ano de 2019, a proporção de FR sobre projeções em relação ao total divulgados foi maior no período de 01/01 a 10/03/2019. Ainda, analisando-se os fatos relevantes que tratam especificamente de projeções, nesse mesmo período de 2020 e o reflexo da COVID-19, verifica-se que a proporção percentual de divulgações com projeções anterior a declaração da situação de crise foi maior (representando $12 \%$ ) do que a proporção no período posterior (10\%).

Entretanto, pela análise de conteúdo dos referidos fatos constata-se que não houve divulgação antecipada com previsão do impacto da COVID-19 nas atividades das empresas analisadas. Considerando que os eventos de projeções divulgados até 11 de março, estavam relacionados em 70\% à "apresentação de projeções de atividades para 2020", mesmo diante da existência de declaração da OMS do surto da doença como Emergência de Saúde Pública de Importância Internacional, em 30 de janeiro do mesmo ano, as empresas não reportaram nenhuma previsão e impacto.

Concentrando a análise nos FR relacionados às projeções, observa-se que $74 \%$ desses ocorreram após a constatação da pandemia, entre 12/03 a 31/03, enquanto que no mesmo 
Mayara Bezerra Barbosa, César Augusto Tibúrcio Silva, Mariana Pereira Bonfim e José Mauro Madeiros Velôso Soares

período de 2019 essa proporção foi de 52\%. Assim, é possível inferir um aumento nos FR relacionados às projeções.

A Azul S.A. foi a primeira empresa a divulgar a suspensão de suas projeções relacionadas a COVID-19, divulgando um documento no dia 12 de março de 2020. 0 documento divulgado pela empresa aérea começa informando que estaria monitorando o impacto da doença nos resultados de 2020, destacando que a "principal prioridade continua sendo a saúde e a segurança de nossos tripulantes e clientes". A seguir, a empresa indica cinco medidas adotadas para reduzir o impacto, com destaque a redução da oferta de voos e negociação de pagamento para preservação de caixa. Ainda no fato relevante, a empresa afirma que "dada a incerteza relacionada com o impacto da propagação do vírus, estamos suspendendo nossas projeções para 2020. Publicaremos uma projeção atualizada assim que tivermos maior visibilidade sobre o impacto do vírus em nossos negócios". Nesta mesma data, o circuit breaker da B3 foi acionado duas vezes, sendo que isto ocorreu seis vezes entre o período de 9 a 18 de março de 2020, afetando os negócios das empresas, influenciando as cotações, a decisão de investidores e a volatilidade do mercado financeiro.

A análise de conteúdo individual dos relatórios de fatos relevantes possibilitou identificar os principais assuntos e contextos relacionados aos termos de pesquisa. 0 conteúdo foi categorizado em 10 grupos de assuntos, e a análise buscou estabelecer uma relação dos fatos e eventos relacionados aos cenários de 2019, sem pandemia, e 2020 com a pandemia da COVID-19, além da temporalidade dos mesmos.

Conforme evidências apresentadas na Tabela 5, os principais assuntos abordados no contexto dos termos foram "suspensão de projeções", seguido pela "atualização das projeções" e "apresentação de projeções" para as atividades do exercício de 2019 e 2020, correspondendo a $82 \%$ e $62,6 \%$, respectivamente, da totalidade de FR sobre projeções.

Tabela 5 - Principais conteúdos dos fatos relevantes sobre previsões

\begin{tabular}{lcc}
\hline Análise de Conteúdo do assunto principal & \multicolumn{2}{c}{$\mathbf{N}^{\circ}$ de FR } \\
\cline { 2 - 3 } Suspensão - projeções & $\mathbf{2 0 2 0}$ & $\mathbf{2 0 1 9}$ \\
Apresentação de projeções de atividades & 35 & 7 \\
Atualização projeções de atividades & 32 & 43 \\
Outros & 34 & 17 \\
Sem projeções ou sem possíveis impactos & 24 & 11 \\
Divulgação e Impactos nos resultados & 15 & - \\
Previsão de retorno das atividades & 9 & 1 \\
Orientações de atividades e projeções & 5 & - \\
Impacto nas projeções de assembleia & 4 & - \\
Manter projeções & 3 & - \\
\hline TOTAL & 2 & $\mathbf{8 1}$ \\
\hline
\end{tabular}

Fonte: elaborada pelos autores (2020).

Dos 81 fatos relevantes sobre projeções divulgados pelas empresas em 2019, constatou-se que no período entre 01/01 e 10/03/2019, 17 FR estavam relacionados à apresentação de projeções, 7 à atualização das projeções de atividades, 5 à suspensão e 4 relacionados a outros assuntos.

Importante destacar que, as suspensões nesse período estavam relacionadas a narrativas de "descontinuidade de divulgação de projeções financeiras (guidance)", por motivos diversos relacionados às empresas, a exemplo da Grendene S.A., em 14/02/2019, e da Pague Menos S.A. que discorrem: 
Projeções nos fatos relevantes das empresas de capital aberto da B3:

impactos da COVID-19

descontinuará a divulgação de metas, projeções e estimativas sobre desempenho futuro (guidance), anteriormente apresentadas em seu Formulário de Referência, em razão da administração entender que esta prática deixou de agregar valor para a Companhia, para seus investidores e o mercado em geral (Grendene S.A.).

Descontinuar a divulgação de projeções e estimativas sobre desempenho futuro (guidance) para o ano de 2019, anteriormente apresentadas em seu Formulário de Referência, em razão das dificuldades da Companhia em obter os documentos e informações necessárias para realização das projeções, bem como em decorrência das alterações no cenário econômico no qual a Companhia se insere (Pague Menos S.A.).

Dessa forma, as companhias demonstram suas dificuldades em cumprirem as características qualitativas da informação contábil, que deve fornecer informações preditivas a seus usuários.

No setor financeiro, que apresentou o maior número de fatos relevantes, observou-se que 11 empresas apresentaram suas projeções para o exercício 2019 entre janeiro a maio, e só identificou 1 suspensão de projeções nos FR, divulgada pela Cielo S.A. em 24/05/2019, no qual a empresa comunica o seguinte trecho:

comunica aos seus acionistas, ao mercado e aos demais interessados [...] que decidiu por descontinuar a projeção (guidance) divulgada em 29 de janeiro de 2019 de lucro líquido entre $R \$ 2,3$ e $R \$ 2,6$ bilhões para o ano de 2019 , sem que sejam estabelecidas novas estimativas (Cielo S.A.).

Dos 163 fatos relevantes relacionados às projeções e estimativas das empresas em 2020, 42 destes foram publicados entre 01/01 e 10/03/2020, sendo 28 relacionados à apresentação de projeções para as atividades de 2020. Desses 28,5 atualizavam projeções anteriores, 2 suspendiam projeções relacionadas a ofertas de ações e os outros 7 se referiam a assuntos diversos. Não foram localizadas referências à COVID-19 nos FR divulgados nesse período.

Em 27 de março, a Eletrobras emitiu um FR informando que manteria as projeções divulgadas. De maneira semelhante a Restoque Comércio e Confecções de Roupas divulgou, em 14 de abril de 2020, que manteria as projeções anteriormente planejadas. Possíveis razões podem estar relacionadas às particularidades do setor de atuação das empresas ou ao otimismo da administração. Mas após 30 dias dessa divulgação, ambas as empresas apresentaram "atualizações de suas projeções".

Após o período de 11/03/2020, foram emitidos 120 novos fatos relevantes, sendo 31 destes sobre suspensões de projeções anteriores, sendo 9 em março, 9 em abril e 8 em maio, 1 em junho e 4 em julho. É relevante destacar que 10 dessas suspensões foram divulgadas por instituições financeiras, sendo que 7 somente em maio que vieram a ser comunicadas aos investidores e demais interessados, referentes aos efeitos da COVID-19 em suas operações.

A empresa Vale S.A., após suspender suas atividades em 16 de março, voltou a atualizálas em 17 e 28 de abril. A companhia manteve-se monitorando os possíveis impactos da COVID-19 e apresentou 4 FR, entre 18/05 e 17/06/2020, informando "não haver alteração no guidance das atividades operacionais da companhia". Em julho, nos dias 20 e 27, a empresa voltou a apresentar atualizações das projeções, destacando que representam uma mera estimativa da administração diante do cenário atual.

As empresas Azul e São Martinho reapresentaram projeções operacionais para as atividades no exercício 2020, respectivamente em 29/06 e 06/07/2020. Ênfase se faz, aos resultados da Azul no primeiro semestre de 2020, no qual a empresa apresentou prejuízo de $\mathrm{R} \$ 2,9$ bilhões. 
Mayara Bezerra Barbosa, César Augusto Tibúrcio Silva, Mariana Pereira Bonfim e José Mauro Madeiros Velôso Soares

Enquanto isso, observa-se que as empresas Cemig e Santander descontinuaram suas projeções somente em 22/07 e 29/07, referentes às projeções anteriormente divulgadas no ano de 2019, nas datas 29/05/2019 e 08/11/2019, respectivamente. Observa-se ainda que, as referidas empresas reiteram o compromisso de manter seus acionistas, credores e o mercado em geral oportunamente informados dos fatos que possam a vir afetar os resultados dessas instituições. No texto do FR mencionado acima, o Banco Santander (Brasil) S.A. informou que:

decidiu descontinuar a divulgação das projeções inicialmente divulgadas por meio de FR datado de 08 de outubro de 2019 [...] motivada pelo atual cenário de incerteza acerca da dimensão e duração da pandemia do COVID-19 e seus impactos no ambiente macroeconômico (Banco Santander S.A.).

Logo, as evidências narrativas demonstram o lapso temporal da instituição ao avaliar os riscos da COVID-19.

Os resultados possibilitam identificar ainda que, nos últimos 20 dias do mês de março de 2020, 14 FR tratavam da impossibilidade relatadas pelas empresas de fazer ou divulgar impactos da COVID-19 nas estimativas e projeções de suas atividades, destacando-se que 5 foram emitidos por empresas do setor de transportes.

Embora a literatura prévia (CEN; CAl, 2013) aborde que em razão da tempestividade a comunicação de perspectivas futuras pode ser possibilitada, os resultados encontrados demonstram ausência do evento pandêmico como justificativa de revisões de projeções acontecidas anterior à declaração pela OMS. Contudo, pelo relevante volume de negociação e investimento com países como a China (GALE; VALDES; ASH, 2019; GOUVEA; MONTOYA, 2013; GOUVEA; KAPELIANIS; LI, 2020; WORLD BANK, 2018) era esperado que, ainda que não fosse declarado como pandêmico, houvesse algum impacto substancial pela interdependência econômica.

Neste ponto, os resultados contribuem para a literatura relacionada a divulgação contábil ao evidenciar a existência de um lapso temporal relativamente expressivo entre a ocorrência de um evento de natureza e dimensionalidade do impacto nos negócios desconhecidos com razoável segurança. Os resultados demonstram que a atribuição de causalidade entre as revisões das projeções e a disseminação do novo coronavírus foram divulgadas por algumas empresas tempestivamente, até no mesmo mês em que foi declarado como pandemia, enquanto outras demoraram até 140 dias para serem anunciadas como fato relevante.

Como é normativamente exigida a divulgação do fato relevante (CVM, 2002) antes de outra veiculação, é possível que na data em que a empresa divulga o evento o mercado já tenha uma expectativa e a formalidade de divulgação funcione como redutora da assimetria de informações, corroborando o pressuposto apresentado por Silva e Fernandes (2009) em relação a este ponto. Neste sentido, o estudo contribui ao destacar que neste cenário potencialmente pessimista analisado, houve relativa demora por algumas empresas até que estas informações, sobre o efeito da doença nas projeções, fossem efetivamente divulgadas.

Apesar da notória diferença de lapso temporal entre as divulgações, não é possível afirmar por meio das evidências encontradas na pesquisa, qual a motivação para que isto acontecesse. Uma implicação prática destes resultados é que o tempo de resposta das empresas pode ser diferente tanto por decisões administrativas que envolvem discricionariedade ou até mesmo pelas limitações de atribuir os impactos de eventos exógenos não previstos nas projeções realizadas. 
Projeções nos fatos relevantes das empresas de capital aberto da B3:

impactos da COVID-19

\section{CONSIDERAÇÕES FINAIS}

Os fatos relevantes são comunicações importantes entre as empresas e o mercado, considerando a expectativa de que tragam informações tempestivas de eventos ocorridos ou relacionados aos negócios que possam impactar decisões. A previsibilidade dos efeitos desses fatos, como da pandemia da COVID-19, que interferem na continuidade das empresas, é uma característica relevante da informação contábil útil e, portanto, deve ser divulgada de forma imediata pelas empresas.

O presente estudo teve como objetivo analisar os reflexos dos primeiros meses da pandemia da COVID-19 no volume de divulgação de fatos relevantes relacionados com as projeções das empresas de capital aberto da B3. Para atender ao propósito da pesquisa, realizou-se uma análise textual dos fatos relevantes divulgados pelas empresas brasileiras de 01 de janeiro à 31 de julho de 2020, comparativamente com os mesmos dados do ano anterior.

As evidências indicaram que a ocorrência da pandemia da COVID-19 ocasionou um aumento no número de fatos relevantes das empresas, em comparação ao ano de 2019 . Os efeitos levaram à suspensão de projeções de atividades e de previsões de resultados e investimentos, decorrentes da crise gerada pelo cenário atual. Constatou-se ainda que a COVID-19 impulsionou setores, como o financeiro e o de transportes, a emitirem o maior número de FR relacionados a COVID-19 e as previsões das atividades operacionais e projeções divulgadas.

Com respeito aos estudos anteriores, os achados dessa pesquisa talvez estejam coerentes com o fator identificado por Marques et al. (2010) que os fatos relevantes emitidos geralmente retratam boas notícias. Os achados indicam que a postergação, ou não divulgação, dos efeitos da pandemia nas projeções pode ser uma maneira de evitar divulgar notícias ruins.

Esses resultados demonstram também que as empresas não disseminaram, em geral, de forma imediata, constatando-se que, proporcionalmente, o maior número de fatos relevantes, relacionados ao impacto da COVID-19 nas projeções das empresas brasileiras, se deram somente nos meses seguintes a decretação pela OMS da doença como pandemia. Este resultado representa uma contribuição prática e profissional da pesquisa. $O$ fato relevante seria o instrumento natural para comunicar a influência de um evento como a pandemia nas projeções. Entretanto, as empresas evitaram comunicar esta informação de maneira tempestiva. Em março de 2020 já era claro que a pandemia traria efeitos nos resultados das empresas, mesmo assim, muitas companhias evitaram divulgar a suspensão das projeções.

O presente estudo avança na análise de previsões da informação contábil construída pelas empresas, decorrentes de cenários como o do novo coronavírus, com observância a tempestividade dos gestores em reportar fatos relevantes que possam impactar a tomada de decisão dos usuários. Apresenta também contribuições à temática ao fornecer um diagnóstico do efeito da crise gerada pela pandemia da COVID-19 nas projeções elaboradas pelas empresas.

Pesquisas adicionais podem analisar outras características dos fatos relevantes, como a existência de conteúdo relacionado a COVID-19 ou o impacto da pandemia nas ações da empresa. Também de forma complementar, pode ser verificado o impacto de outras divulgações não voluntárias emitidas pelas companhias, como as publicadas em redes sociais institucionais, ligadas ao novo coronavírus. 
Mayara Bezerra Barbosa, César Augusto Tibúrcio Silva, Mariana Pereira Bonfim e José Mauro Madeiros Velôso Soares

\section{REFERÊNCIAS}

ARGILÉS-BOSCH, J. M.; MIARONS, M.; GARCIA-BLANDON, J.; BENAVENTE, C.; RAVENDA, D. Usefulness of fair valuation of biological assets for cash flow prediction. Spanish Journal of Finance and Accounting, v. 47, n. 2, p. 157-180, 2018. DOI:

https://doi.org/10.1080/02102412.2017.1389549.

BARDIN, L. Análise de Conteúdo. São Paulo: Edições 70, 2016.

BERNARDES, J. R.; NASCIMENTO, J. C. H. B.; AYRES, R. M.; SIQUEIRA, J. R. M. Legibilidade dos fatos relevantes: uma análise na Vale SA no período de agosto 2012 a agosto de 2016. Pensar Contábil, v. 20, n. 71, 2018. Disponível em: http://www.atena.org.br/revista/ojs2.2.3-06/index.php/pensarcontabil/article/view/3316. Acesso em: 08 ago. 2020.

BROWN, N. Going Public: the benefits and pitfalls of non-GAAP metrics. Revista de Educação e Pesquisa em Contabilidade (REPeC), v. 14, n. 2, 2020. DOI:

https://doi.org/10.17524/repec.v14i2.2670.

BROWN, P. The ValueReporting Revolution: Moving Beyond the Earnings Game: Robert G. Eccles, Robert H. Herz, E. Mary Keegan and David MH Phillips, Wiley, New York, 2001, xvii+ 349 pp. The International Journal of Accounting, v. 37, n. 1, p. 145-148, 2002. DOI: https://doi.org/10.1016/s0020-7063(02)00130-9.

CEN, Z.; CAI, R. 'Impression management' in Chinese corporations: a study of chairperson's statements from the most and least profitable Chinese companies. Asia Pacific Business Review, v. 19, n. 4, p. 490-505, 2013. DOI: https://doi.org/10.1080/13602381.2013.811825.

COMITÊ DE PRONUNCIAMENTOS CONTÁBEIS. PRONUNCIAMENTO TÉCNICO CPC 00. Estrutura Conceitual para Relatório Financeiro. Brasília, dez., 2019. Disponível em: http://static.cpc.aatb.com.br/Documentos/573_CPC00(R2).pdf. Acesso em: 08 ago. 2020.

COMISSÃO DE VALORES MOBILIÁRIOS. OFÍCIO-CIRCULAR/CVM/SNC/SEP/no 358. Brasília, jan., 2002. Disponível em: http://www.cvm.gov.br/legislacao/instrucoes/inst358.html. Acesso em: 10 mar. 2020.

COMISSÃO DE VALORES MOBILIÁRIOS. OFÍCIO-CIRCULAR/CVM/SEP/no 02. Rio de Janeiro, fev., 2016. Disponível em: http://www.cvm.gov.br/export/sites/cvm/legislacao/oficioscirculares/sep/anexos/oc-sep-0216.pdf. Acesso em: 21 nov. 2020.

DANTAS, J. A.; ZENDERSKY, H. C.; SANTOS, S. C.; NIYAMA, J. K. A dualidade entre os benefícios do disclosure e a relutância das organizações em aumentar o grau de evidenciação. Revista Economia \& Gestão, v. 5, n. 11, p. 56-76, 2005.

DELOITTE. Impactos financeiros da Covid-19: como lidar com crises e volatilidades. 2020. Disponível em: https://www2.deloitte.com/br/pt/pages/finance/articles/impactosfinanceiros-covid-19.html. Acesso em: 08 ago. 2020 
Projeções nos fatos relevantes das empresas de capital aberto da B3: impactos da COVID-19

DENNIS, D.; SEERY, A. IFRS accounting considerations of the Covid-19 outbreak. EYGM Limited. Irlanda, mar., 2020. Disponível em: https://www.ey.com/en_ie/accountingchange/ifrs-accounting-considerations-of-the-covid-19-outbreak. Acesso em: 08 ago. 2020.

DOVERN, J.; JANNSEN, N. Systematic errors in growth expectations over the business cycle. International Journal of Forecasting, v. 33, n. 4, p. 760-769, 2017. DOI: https://doi.org/10.1016/j.ijforecast.2017.03.003.

ESPÍNDULA, R. P.; COSTA, P. S. Análise da influência da publicação e da legibilidade dos atos ou fatos relevantes no preço da ação das empresas brasileiras de capital aberto no período de 2002 a 2006. In: Seminário de Iniciação Científica, 12., 2008, Uberlândia. Anais eletrônicos [...]. Uberlândia: UFU, 2008. Disponível em: https://ssl4799.websiteseguro.com/swge5/seg/cd2008/PDF/IC2008-0260.PDF. Acesso em: 08 ago. 2020.

FERREIRA, D. S. B. Volume de negociação, fatos relevantes e notícias de imprensa econômica no Brasil. Contabilidade, Gestão e Governança, v. 13, n. 3, p. 53-70, 2010. Disponível em: https://www.revistacgg.org/contabil/article/view/285. Acesso em: 08 ago. 2020.

FORBES, K. J.; RIGOBON, R. No contagion, only interdependence: measuring stock market comovements. Journal of Finance, v. 57, n. 5, p. 2223-2261, 2002. DOI: https://doi.org/10.1111/0022-1082.00494.

GALE, F.; VALDES, C.; ASH, M. Interdependence of China, United States, and Brazil in Soybean Trade. New York: US Department of Agriculture's Economic Research Service (ERS) Report, p. 1-48, 2019.

GOUVEA, R.; MONTOYA, M. Brazil \& China: partners or competitors? Designing strategic alliances in the age of uncertainty. Asian Journal of Latin American Studies, v. 26, n. 1, p. 123, 2013. DOI: http://doi.org/10.22945/ajlas.2013.26.1.1.

GOUVEA, R.; KAPELIANIS, D.; LI, S. Fostering intra-BRICS trade and investment: The increasing role of China in the Brazilian and South African economies. Thunderbird International Business Review, v. 62, n. 1, p. 17-26, 2020. DOI: https://doi.org/10.1002/tie.22098.

HRIBAR, P.; YANG, H. CEO overconfidence and management forecasting. Contemporary Accounting Research, v. 33, n. 1, p. 204-227, 2015. DOI: https://doi.org/10.1111/19113846.12144 .

INTERNATIONAL MONETARY FUND. The great lockdown: worst economic downturn since the great depression. Estados Unidos, mar., 2020. Disponível em: https://www.imf.org/en/News/Articles/2020/03/23/pr2098-imf-managing-directorstatement-following-a-g20-ministerial-call-on-the-coronavirus-emergency. Acesso em: 08 ago. 2020. 
Mayara Bezerra Barbosa, César Augusto Tibúrcio Silva, Mariana Pereira Bonfim e José Mauro Madeiros Velôso Soares

IOANNIDIS, J. P. A. Coronavirus disease 2019: the harms of exaggerated information and non-evidence-based measures. European Journal of Clinical Investigation, v. 50, n. 4, p. 1-5, 2020. DOI: https://doi.org/10.1111/eci.13222.

IOANNIDIS, J.; CRIPPS, S.; TANNER, M. Forecasting for COVID-19 has failed. International Journal of Forecasting, p. 1-16, 2020. DOI: https://doi.org/10.1016/j.ijforecast.2020.08.004.

ISMAILESCU, I.; KAZEMI, H. Contagion or interdependence in emerging debt markets. The Banking and Finance Review, v. 3, n. 2, p. 1-15, 2011. Disponível em: http://webpage.pace.edu/iismailescu/Papers/ContagionPaper_IK.pdf. Acesso em: 08 ago. 2020.

KOS, S. R.; BARROS, C. M. E.; COLAUTO, R. D. Impacto da divulgação de eventos subsequentes no retorno anormal: Estudo em Companhias do Ibovespa. Revista Ambiente Contábil, v. 9, n. 2, p. 60-79, 2017. Disponível em:

https://periodicos.ufrn.br/ambiente/article/view/9896. Acesso em: 08 ago. 2020.

LIMA, N. T.; BUSS, P. M.; PAES-SOUSA, R. A pandemia de COVID-19: uma crise sanitária e humanitária. Cadernos de Saúde Pública, v. 36, n. 7, 2020. DOI:

https://doi.org/10.1590/0102-311x00177020.

MARQUES, V. A.; LARA, C. O.; AYALA, L. C.; LAMOUNIER, W. M. O Impacto dos Fatos Relevantes no Mercado de Capitais: um estudo dos fatos publicados pelas empresas participantes do Novo Mercado do segmento Bovespa. Sociedade, Contabilidade e Gestão, v. 5, n. Especial, 2010. DOI: https://doi.org/10.21446/scg_ufrj.v5i3.13218.

McKIBBIN, W. J.; FERNANDO, R. The Global Macroeconomic Impacts of COVID-19: Seven Scenarios (March 2, 2020). CAMA Working Paper $n^{\circ}$ 19/2020. DOI: https://doi.org/10.2139/ssrn.3547729.

MIIHKINEN, A. The usefulness of firm risk disclosures under different firm riskiness, investorinterest, and market conditions: New evidence from Finland. Advances in Accounting, v. 29, n. 2, p. 312-331, 2013. DOI: https://doi.org/10.1016/j.adiac.2013.09.006.

NIYAMA, J. K.; SILVA, C. A. T. Teoria da Contabilidade. São Paulo: Atlas, 2013.

ROVER, S.; MURCIA, F. D.; BORBA, J. A.; VICENTE, E. F. R. Divulgação de informações ambientais nas demonstrações contábeis: um estudo exploratório sobre o disclosure das empresas brasileiras pertencentes a setores de alto impacto ambiental. Revista de Contabilidade e Organizações, v. 2, n. 3, p. 53-72, 2008. DOI: https://doi.org/10.11606/rco.v2i3.34713.

ROVER, S.; BORBA, J. A.; MURCIA, F. D. Características do disclosure ambiental de empresas brasileiras potencialmente poluidoras: análise das demonstrações financeiras e dos relatórios de sustentabilidade do período de 2005 a 2007. Contextus Revista Contemporânea de Economia e Gestão, v. 7, n. 1, p. 23-36, 2009. DOI: https://doi.org/10.19094/contextus.v7i1.32101. 
Projeções nos fatos relevantes das empresas de capital aberto da B3: impactos da COVID-19

SALOTTI, B. M.; YAMAMOTO, M. M. Divulgação voluntária da demonstração dos fluxos de caixa no mercado de capitais brasileiro. Revista Contabilidade \& Finanças, v. 19, n. 48, p. 3749, 2008. DOI: https://doi.org/10.1590/s1519-70772008000300004.

SANTOS, J. G. C.; COELHO, A. C. Value-relevance do disclosure: fatores e gestão de riscos em firmas brasileiras. Revista Contabilidade \& Finanças, v. 29, n. 78, p. 390-404, 2018. DOI: https://doi.org/10.1590/1808-057x201806150.

SENA, B. H. S.; SILVA, C. A. T.; ARRIAL, R. T. Classificação do conteúdo de documentos contábeis usando aprendizagem de máquina: o caso dos fatos relevantes. Revista de Educação e Pesquisa em Contabilidade, v. 4, n. 2, p. 23-42, 2010. DOI: https://doi.org/10.17524/repec.v4i2.199.

SILVA, C. A. T.; FELIPE, E. S. Avaliação da influência de textos narrativos de fatos relevantes no preço das ações de empresas brasileiras. Revista Contabilidade e Controladoria, v. 2, n. 2, 2010. DOI: http://dx.doi.org/10.5380/rcc.v2i2.19460.

SILVA, C. A. T.; FERNANDES, J. L. T. Legibilidade dos fatos relevantes no Brasil. Revista de Administração Contemporânea - RAC Eletrônica, v. 3, n. 1, p. 142-159, 2009. Disponível em: http://www.anpad.org.br/periodicos/arq_pdf/a_818.pdf. Acesso em: 08 ago. 2020.

SILVA, A.; SOUZA, T. R.; KLANN, R. C. Tempestividade da informação contábil em empresas familiares brasileiras. Revista de Administração de Empresas, v. 56, n. 5, 2016. DOI: https://doi.org/10.1590/s0034-759020160504.

SINGH, A.; SINGH, M. Conditional co-movement and dynamic interactions: US and BRIC equity markets. Economic Annals, v. 62, n. 212, p. 85-111, 2017. DOI: https://doi.org/10.2298/eka1712085s.

VERRECCHIA, R. E. Essays on disclosure. Journal of Accounting and Economics, v. 32, n. 1-3, p. 97-180, 2001. DOI: https://doi.org/10.1016/s0165-4101(01)00025-8.

WORLD BANK. Brazil exports, imports and trade balance by country 2018. World Integrated Trade Solution (WITS). 2018. Disponível em: https://wits.worldbank.org/CountryProfile/en/Country/BRA/Year/LTST/TradeFlow/EXPIMP/ Partner/by-country. Acesso em: 06 ago. 2020. 